\title{
Epidermal Growth Factor Mutation as a Diagnostic and Therapeutic Target in Metastatic Poorly Differentiated Thyroid Carcinoma: A Case Report and Review of the Literature
}

\author{
Hazel Lote Jaishree Bhosle Khin Thway Kate Newbold \\ Mary O'Brien \\ Royal Marsden Hospital, Sutton, Surrey, UK
}

\section{Key Words}

Metastatic thyroid carcinoma - Poorly differentiated thyroid carcinoma - Targeted therapy . Epidermal growth factor mutation · Tyrosine kinase inhibitors · Erlotinib · Intratumoural heterogeneity $\cdot$ Poorly differentiated carcinoma

\begin{abstract}
Poorly differentiated cancers are a diagnostic and therapeutic challenge in oncology. New therapies are needed for patients with poorly differentiated thyroid carcinoma (PDTC) or anaplastic thyroid cancer, as these patients often present with advanced disease and effective systemic treatment options are currently limited. Epidermal growth factor (EGFR) mutations may occur in PDTC more often than previously thought. However, there are fewer than 6 cases reported in the literature where EGFR tyrosine kinase inhibitors (TKIs) (such as erlotinib or gefitinib) were used to target EGFR mutations in PDTC. Here, we present the case of a 79year-old male with metastatic PDTC with an EGFR mutation who responded to treatment with the selective EGFR TKI erlotinib, with a progression-free survival of more than 11 months. A lung primary rather than a thyroid primary was initially detected. We suggest that the EGFR status should be analysed at diagnosis in any patient with a poorly differentiated tumour. The presence of an EGFR mutation may provide an effective therapeutic pathway for these patients. This pathway requires further investigation and consideration in the future.
\end{abstract}




\section{Introduction}

Poorly differentiated cancers usually behave more aggressively and are associated with worse survival rates than well-differentiated cancers [1]. They therefore represent an oncologic therapeutic challenge.

Poorly differentiated thyroid carcinoma (PDTC) is a term coined in the 1980s. PDTC lies on the spectrum between well-differentiated and anaplastic thyroid carcinoma [1], it accounts for only $4-7 \%$ of thyroid cancers worldwide and is frequently advanced or metastatic at the time of diagnosis [1]. With less differentiation, the expression of the sodium iodide symporter is lost and therefore, the utility of radioiodine as a therapeutic option is reduced as the tumour becomes iodide non-avid [1]. The evidence for external beam radiotherapy is less robust and standard chemotherapy agents are not useful [1]. New targeted therapies are needed for patients with PDTC, as these patients typically develop advanced iodine refractory disease [1].

Targeted therapies are becoming increasingly important in the management of PDTC. The most common mutations in PDTC are RAS, p53 and BRAF mutations [2]. RET mutations in PDTC and undifferentiated thyroid and lung cancers are rare [2, 3]. Sorafenib is a multikinase inhibitor targeting RAS, BRAF/MEK/ERK signaling pathways, ligandindependent RET/PTC receptor tyrosine kinase activation, VEGF and platelet-derived growth factor (PDGF) pathways [4]. Phase 3 data from the DECISION trial, recently presented at ASCO and published in Lancet, has led to sorafenib becoming the standard 1st line medication for the treatment of iodine refractory thyroid cancer [4]. This trial compared sorafenib versus placebo in iodine refractory thyroid cancer and the results demonstrated a progression free survival (PFS) advantage of 5 months in the sorafenib group (10.8 months in the sorafenib cohort vs. 5.8 months in the placebo group) [4].

Combrestatin A-4 phosphate, also known as fosbretabulin (CA4P), is a vascular disrupting agent that acts by binding to the beta-subunit of tubulin [5]. The FACT trial explored its efficacy in the treatment of anaplastic thyroid cancer [5]. This trial was a prospective randomised controlled phase $2 / 3$ trial assessing the safety and the efficacy of carboplatin/paclitaxel with CA4P versus without CA4P [5]. Eighty patients were enrolled and the trial closed due to poor accrual. There was no statistically significant improvement in survival with CA4P [5].

Epidermal growth factor (EGFR) mutations as therapeutic targets are well-established in the treatment of metastatic lung adenocarcinoma [6]. Erlotinib was licensed in 2011 for the 1st line treatment in patients with metastatic lung adenocarcinoma who harbour EGFR mutations [6]. The BATTLE trial, a phase 3 trial exploring the use of sorafenib in the 3rd line setting for NSCLC patients, did not demonstrate any improvement in overall survival and has therefore not been published. However, subgroup analysis presented at ESMO in 2013 suggested that patients with EGFR mutations might benefit from sorafenib.

The incidence of EGFR mutations in thyroid carcinoma was previously thought to be low [7]. However, more recently, it has been suggested that EGFR mutations may be in the region of 30\% [7]. A retrospective examination of thyroid tissue from a series of 23 patients with papillary thyroid carcinoma was striking: 7 were found to harbour drug-sensitising mutations and 1 patient had EGFR amplification [7]. This suggests that EGFR mutations may occur within a certain subset of thyroid carcinoma patients, just as EGFR mutations in lung carcinoma occur within subsets of patients (adenocarcinoma, never-smokers, young, female, Asian) [7]. Additionally, intratumoral heterogeneity may influence the frequency that these mutations are detected and may explain why initial studies suggested that EGFR mutations were rare $[7,8]$. 
Phase 2 trials evaluating the use of Gefitinib in patients with advanced thyroid cancer did not select patients based on EGFR mutations $[9,10]$. However, although these phase 2 trials did not show positive results, 1 patient within a trial responded for more than 12 months $[9,10]$. A literature search has revealed fewer than 6 cases in the literature of patients with EGFR-mutated thyroid carcinoma, or lung cancer with thyroid metastases, who have been treated with EGFR TKIs (erlotinib or gefitinib). All of these reported cases responded to EGFR TKI treatment $[7,9,11-14]$.

Hogan et al. [14] presented the case of a patient with an oncocytic, focally anaplastic thyroid cancer treated with a therapeutic trial of erlotinib. They report that the patient had a dramatic response to erlotinib [14]. Mutational analysis revealed mutations within exons 18 and 20 of the EGFR gene [14]. Interestingly, in lung adenocarcinoma, exon 18 and 20 mutations confer relative resistance to TKI therapy [15].

Here, we present the case of a 79-year-old male with metastatic PDTC with an EGFR mutation who was treated with the TKI erlotinib. Our patient had a good partial response to treatment with a PFS of 11 months. We suggest that targeted therapy with an EGFR TKI is a possible targeted treatment pathway for patients with metastatic thyroid carcinoma who harbour an EGFR mutation.

\section{The Case}

A 79-year-old Caucasian presented with a 3-week history of right hip pain. Hip X-ray demonstrated a lytic lesion within the right pubic ramus. Biopsy of this lesion showed metastatic TTF1-positive adenocarcinoma of either lung or thyroid origin, therefore the patient was referred to the lung cancer team at the Royal Marsden Hospital. The patient was otherwise asymptomatic with a performance status of 1 . Past medical history and family history were unremarkable. He was a previous light smoker with a 3-pack-per-year history in total.

An FDG-PET CT scan showed a strongly FDG-positive (SUV 16.57) lesion in the right inferior pubic ramus, consistent with a metastatic deposit. No other suspicious bony lesions were identified. There was high-grade irregular increased FDG uptake involving both lobes of the thyroid gland (SUV max 16.95). There were multiple FDG positive lymph nodes involving the right supraclavicular, right thoracic inlet, paratracheal, subcarinal, right hilar, anterior mediastinal and posterior mediastinal regions in keeping with nodal metastases. There was some small pleural-based nodularity within the apical segment of the right lower lobe, but no discrete pulmonary lesions were identified. Overall, these PET CT appearances were highly suggestive of stage IV metastatic thyroid cancer (bilateral) with extensive nodal and solitary bone metastases.

The patient was initially treated with zolendronic acid and palliative radiotherapy (20 Gray in 5 fractions) to the symptomatic metastasis in the right pubic ramus. The Royal Marsden Hospital histopathology review of the pubic ramus biopsy showed adenocarcinoma positive for TTF1 and CK7, and negative for CK20 and PSA. Mutational analysis revealed 2 mutations within the EGFR gene: c.2573T >G; p.L858R and EGFR exon 20 insertion. Thyroglobulin and calcitonin immunohistochemistry were negative. The pathologist concluded that the primary was likely thyroid (fig. 1).

Fine-needle aspiration of the thyroid confirmed PDTC. Serum thyroglobulin was 176. The patient's low thyroglobulin value despite the large-volume disease may reflect the patient's poorly differentiated disease that no longer expresses thyroglobulin. This would fit with the recognised 'flip-flop' phenomenon: as DTC becomes PDTC, the FDG uptake on PET 
imaging increases, whilst the radioiodine uptake decreases. The proposed management was total thyroidectomy followed by radioiodine (RAI) therapy in the first instance.

Prior to the planned thyroidectomy, the patient was found to have a DVT and PEs and was commenced on therapeutic low-molecular-weight heparin. Thyroidectomy was postponed whilst anticoagulation was established. In the interim, in view of the known EGFR mutation, the patient was commenced on systemic treatment with the EGFR TKI erlotinib at a dose of $150 \mathrm{mg}$ once a day. Six weeks later, he underwent total thryroidectomy with right level 4/5 (supraclavicular) and left level 3 neck dissection. Final histopathology demonstrated a poorly differentiated carcinoma with sarcomatoid areas, staged as pT4a pN1b cM1 (fig. 2). Mutational analysis of the thyroid specimen showed an EGFR mutation c.2573T $>$ G; p.Leu858Arg (p.L858R) (a sensitising mutation) and did not demonstrate an EGFR exon 20 insertion or mutation. There were no mutations in BRAF, KRAS or ALK.

Post-operatively, the patient continued on $150 \mathrm{mg}$ of erlotinib once a day. A repeat FDG PET scan performed 9 months after initial diagnosis demonstrated an ongoing good partial response (fig. 3). An iodine uptake scan performed 11 months after diagnosis showed ongoing PFS, with no iodine-131 uptake in the mediastinal nodal or bony disease.

The patient has not been treated with RAI in view of the response to erlotinib, the tolerability of the full dose (150 mg once a day), the minimal side effects from erlotinib and the patient's good quality of life. After 12 months of erlotinib therapy, PET imaging suggests that the patient's disease is beginning to progress. However, he continues on erlotinib at present.

\section{Discussion}

We have presented the case of a 79-year-old male with metastatic PDTC with an EGFR mutation who responded to treatment with the EGFR TKI erlotinib. The clinical decision not to treat him with RAI was based upon the evidence that there was high FDG uptake on PET, low serum thyroglobulin and a negative diagnostic iodine scan. Taken together, this suggested that this patient's disease would be less likely to respond to RAI. Additionally, the patient was already responding to erlotinib at that stage, and had minimal side effects from treatment.

Our patient's bone metastasis contained 2 EGFR mutations: c.2573T $>$ G; p.Leu858Arg (p.L858R) and p.Exon 20 Insertion. Interestingly, mutational analysis on the thyroid specimen post-thyroidectomy revealed only a single EGFR mutation: c.2573T $>$ G; p.Leu858Arg (p.L858R). The literature suggests that exon 20 mutations may confer resistance to EGFR TKIs [15], and the discrepancy between these 2 sites, plus the fact that the patient clinically responded to erlotinib, supports intratumoral heterogeneity [8].

Our patient's PFS of 11 months on erlotinib was comparable to the DECISION data showing a PFS of 10.8 months on sorafenib [4]. On further review of comparable literature, we found that there are only 6 similar clinical cases reported: 5 of these are cases of patients with disease in both the lung and the thyroid, and 1 of these is thyroid-only disease [7, 9, 1114]. Different authors drew different conclusions regarding whether the primary is thyroid or lung; this will be discussed in detail below. Some of the authors concluded that distant metastases were lung primary with thyroid metastases (instead of thyroid primary with lung metastases) based on the presence of EGFR mutations [11,13].

The first of the similar case reports is from Masago et al. [7]. Their patient was originally thought to have lung adenocarcinoma, but was subsequently found to have advanced papillary thyroid carcinoma. This patient had an activating EGFR mutation (exon 19 mutation) and responded to the EGFR inhibitor gefitinib [7]. Another case presented by the 
same group at ASCO in 2011 demonstrated a similar clinical picture: a patient with metastatic anaplastic thyroid carcinoma with an exon 21 mutation who responded to first line erlotinib and survived for more than 6 months [9]. Interestingly, this patient's EGFR mutation was present at the metastatic sites (transbronchial and cervical lymph node biopsies), but not in the thyroid primary [9]. This further supports the case for intratumoural heterogeneity, with mutations occurring at the metastatic sites, but not the primary [8]. A similar picture of mutational heterogeneity was found in our patient.

In 2011, Albany et al. [11] presented the case of a patient who presented with synchronous lung and thyroid masses, both of which were biopsied. The thyroid biopsy was TTF1 and CK7 positive, and the authors state that the lung biopsy revealed 'adenocarcinoma of the lung. The authors felt that the thyroid biopsy could not distinguish whether the thyroid mass was a lung metastasis or a thyroid primary. However, on the basis of the presence of an EGFR exon 21 mutation in the lung biopsy, Albany et al. concluded that this must be a lung primary with a thyroid metastasis. The patient was treated with first line erlotinib and achieved a response which lasted for more than 8 months. The authors do not state whether the thyroid biopsy was tested for EGFR mutations. This case may therefore possibly represent a case of metastatic thyroid carcinoma harbouring an EGFR mutation responding to erlotinib. Such a case would be similar to our patient's case [11].

Matsumoto et al. [12] describe a 56-year-old female - previously radically treated for lung cancer - who, a few months later, was found to have a distant lung metastasis in addition to a papillary thyroid carcinoma (detected synchronously). EGFR mutational analysis was performed on the lung biopsy obtained from the new lung metastasis, and the patient was found to have an exon 19 mutation. The patient was treated systemically with gefitinib and demonstrated a complete response in both the lung and the thyroid [12]. The authors do not state whether EGFR mutational analysis was performed on the thyroid again, this clinical picture could represent a thyroid primary harbouring an EGFR mutation.

Hashimoto et al. [13] describe a 60-year-old female with adenocarcinoma in both the lung and the thyroid. Within the thyroid, there were 2 distinct areas of carcinoma within a single nodule. One area was found to harbour an EGFR exon 21 mutation whilst the other area was not. The mass within the lung also harboured an exon 21 mutation. The group concluded that a lung adenocarcinoma had metastasised to the thyroid and the patient was treated with a TKI targeting EGFR [13]. This case bears similarity to the cases presented by Matsumoto et al. and Albany et al. [11, 12].

\section{Conclusion}

Here we have presented the case of a Caucasian male with EGFR-mutated metastatic thyroid carcinoma, treated with $150 \mathrm{mg}$ of erlotinib. He had a sustained response for 11 months, experienced minimal side effects from treatment, and maintained an excellent quality of life.

In summary, our case, taken together with the available literature, suggests that EGFR mutations can and do occur in thyroid carcinomas. In some of the examined case reports discussed here, some authors concluded that patients had primary lung carcinoma with thyroid metastases based on the presence of EGFR mutations. We suggest that some of these cases may represent primary thyroid carcinoma with metastatic sites harbouring EGFR mutations. In view of intratumoural heterogeneity, it may be beneficial to perform mutational analysis using biopsies from both primary and metastatic sites. 
In the era of targeted therapy, targeting the EGFR receptor in metastatic thyroid carcinoma represents a change in the way this cancer can be treated. We suggest that mutational analysis is performed at diagnosis for patients with poorly differentiated carcinomas. We conclude that treatment with an EGFR TKI in EGFR-mutant metastatic thyroid carcinoma may be a valuable treatment pathway.

\section{References}

1 Hannallah J, Rose J, Guerrero MA: Comprehensive literature review: recent advances in diagnosing and managing patients with poorly differentiated thyroid carcinoma. Int J Endocrinol 2013;2013:317487.

-2 Soares P, Lima J, Preto A, et al: Genetic alterations in poorly differentiated and undifferentiated thyroid carcinomas. Curr Genomics 2011;12:609-617.

-3 D'Arcangelo M, D’Incecco A, Cappuzzo F: Rare mutations in non-small-cell lung cancer. Future Oncol 2013;9:699-711.

-4 Brose MS, Nutting CM, Jarzab B, et al: Sorafenib in radioactive iodine-refractory, locally advanced or metastatic differentiated thyroid cancer: a randomised, double-blind, phase 3 trial. Lancet 2014, Epub ahead of print.

5 Sosa JA, Balkissoon J, Lu SP, et al: Thyroidectomy followed by fosbretabulin (CA4P) combination regimen appears to suggest improvement in patient survival in anaplastic thyroid cancer. Surgery 2012;152:10781087.

-6 Zhou C, Wu YL, Chen G, et al: Erlotinib versus chemotherapy as first-line treatment for patients with advanced EGFR mutation-positive non-small-cell lung cancer (OPTIMAL, CTONG-0802): a multicentre, open-label, randomised, phase 3 study. Lancet Oncol 2011;12:735-742.

7 Masago K, Asato R, Fujita S, et al: Epidermal growth factor receptor gene mutations in papillary thyroid carcinoma. Int J Cancer 2009;124:2744-2749.

8 Gerlinger M, Rowan AJ, Horswell S, et al: Intratumor heterogeneity and branched evolution revealed by multiregion sequencing. N Engl J Med 2012;366:883-892.

-9 Masago K, Miura M, Toyama Y, et al: Good clinical response to erlotinib in a patient with anaplastic thyroid carcinoma harboring an epidermal growth factor somatic mutation, L858R, in exon 21. J Clin Oncol 2011;29:e465-e467.

10 Pennell NA, Daniels GH, Haddad RI, et al: A phase II study of gefitinib in patients with advanced thyroid cancer. Thyroid 2008;18:317-323.

11 Albany C, Jain A, Ulbright TM, Einhorn LH: Lung cancer, thyroid cancer or both: an unusual case presentation. J Thorac Dis 2011;3:271-273.

12 Matsumoto F, Itoh S, Ohba S, Ikeda K: A case report of thyroid cancer showing a remarkable effect of gefitinib. Auris Nasus Larynx 2009;36:623-626.

13 Hashimoto K, Yamamoto H, Nakano T, et al: Tumor-to-tumor metastasis: lung adenocarcinoma metastasizing to a follicular variant of papillary thyroid carcinoma. Pathol Int 2011;61:435-441.

14 Hogan T, Jing Jie Yu, Williams HJ, et al: Oncocytic, focally anaplastic, thyroid cancer responding to erlotinib. J Oncol Pharm Pract 2009;15:111-117.

15 Gazdar AF: Activating and resistance mutations of EGFR in non-small-cell lung cancer: role in clinical response to EGFR tyrosine kinase inhibitors. Oncogene 2009;28(suppl 1):S24-S31. 


\section{Case Reports in Oncology}

Case Rep Oncol 2014;7:393-400

Lote et al.: EGFR Mutation as a Diagnostic and Therapeutic Target in Metastatic PDTC: A Case Report and Review of the Literature

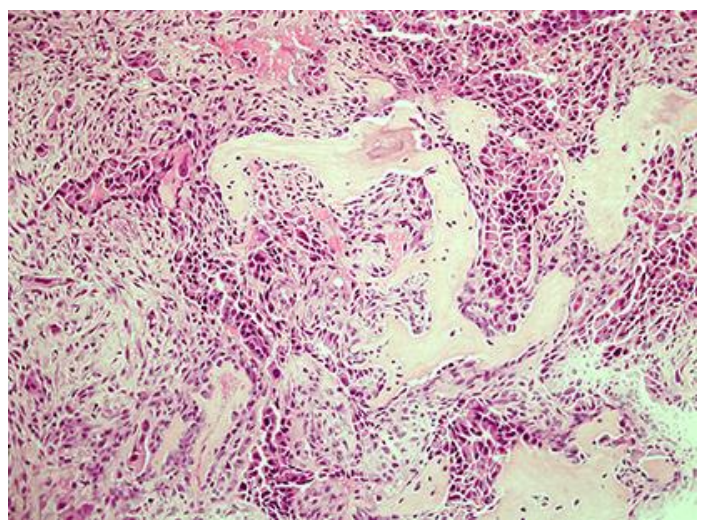

Fig. 1. This biopsy from the pubic ramus shows extensive tumour infiltrating and destroying of the bone (centre). The tumour is composed of nests and trabeculae of columnar cells, focally with glandular formations (left of field). The morphology and immunoprofile (of CK7 and TTF1 positivity) are consistent with adenocarcinoma, in keeping with metastasis from the thyroid. (HE. ×200).

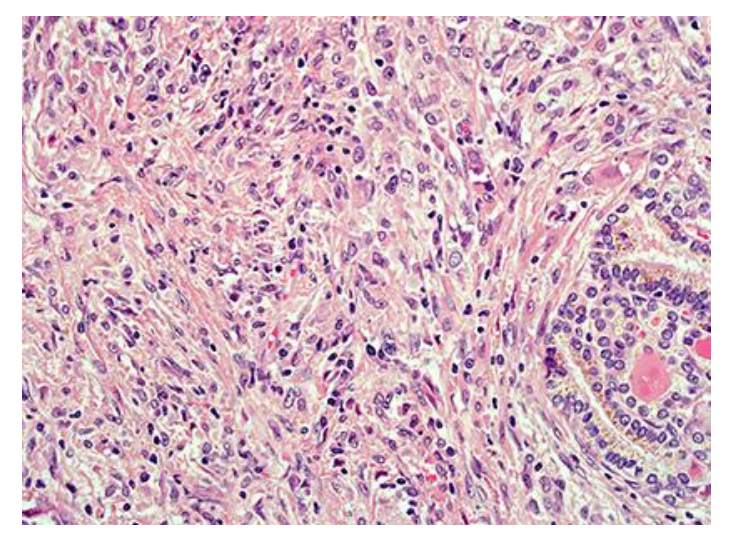

Fig. 2. The thyroidectomy specimen shows extensive, infiltrative cellular tumour composed of discohesive, moderately to markedly pleomorphic, epithelioid and spindle cells, largely in a patternless distribution. There are some entrapped glandular structures (bottom right of field). The features are consistent with poorly differentiated adenocarcinoma with prominent sarcomatoid areas (HE. $\times 200)$. 


\section{Case Reports in Oncology}

\begin{tabular}{l|l}
\hline Case Rep Oncol 2014;7:393-400 \\
\hline DOI: $10.1159 / 000364856$ & $\begin{array}{l}\text { ○ 2014 S. Karger AG, Basel } \\
\text { www.karger.com/cro }\end{array}$ \\
\hline
\end{tabular}

Lote et al.: EGFR Mutation as a Diagnostic and Therapeutic Target in Metastatic PDTC: A Case Report and Review of the Literature

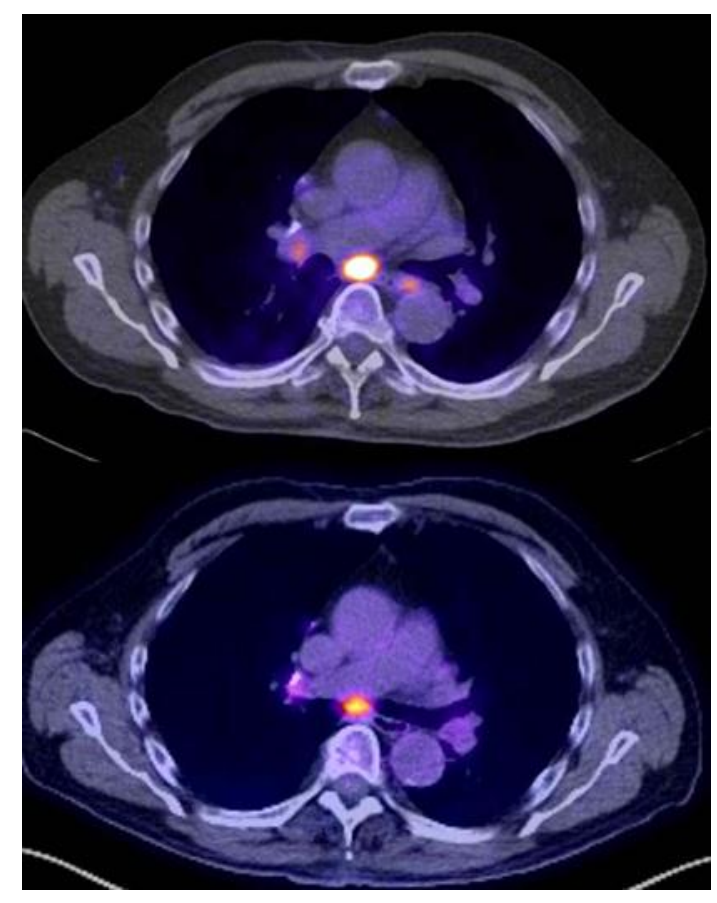

Fig. 3. The upper PET-CT image shows the cross-sectional appearances in January 2013, prior to initiation of erlotinib. The lower PET-CT image demonstrates the good partial response seen in September 2013, after 9 months of erlotinib, with improvement in the mediastinal and hilar lymphadenopathy. 